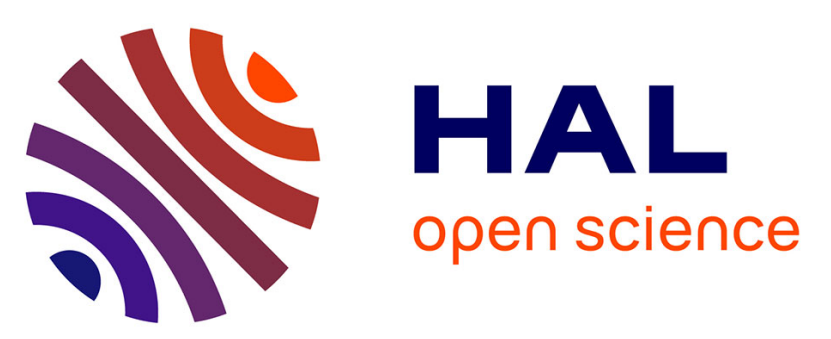

\title{
Elucidation of non-intentionally added substances migrating from polyester-polyurethane lacquers using automated LC-HRMS data processing
}

Elsa Omer, Ronan Cariou, Gerald Remaud, Yann Guitton, Helene Germon, Paul Hill, Gaud Dervilly-Pinel, Bruno Le Bizec

\section{To cite this version:}

Elsa Omer, Ronan Cariou, Gerald Remaud, Yann Guitton, Helene Germon, et al.. Elucidation of non-intentionally added substances migrating from polyester-polyurethane lacquers using automated LC-HRMS data processing. Analytical and Bioanalytical Chemistry, 2018, 410 (22), pp.5391-5403. 10.1007/s00216-018-0968-z . hal-02140927

\section{HAL Id: hal-02140927 \\ https://hal.science/hal-02140927}

Submitted on 27 May 2020

HAL is a multi-disciplinary open access archive for the deposit and dissemination of scientific research documents, whether they are published or not. The documents may come from teaching and research institutions in France or abroad, or from public or private research centers.
L'archive ouverte pluridisciplinaire HAL, est destinée au dépôt et à la diffusion de documents scientifiques de niveau recherche, publiés ou non, émanant des établissements d'enseignement et de recherche français ou étrangers, des laboratoires publics ou privés. 


\title{
Elucidation of non-intentionally added substances migrating from polyester-polyurethane lacquers using automated LC-HRMS data processing
}

\author{
Elsa Omer ${ }^{1,2,3} \cdot$ Ronan Cariou $^{1} \cdot$ Gérald Remaud $^{2} \cdot$ Yann Guitton $^{1} \cdot$ Hélène Germon $^{3} \cdot$ Paul Hill $^{3}$ - Gaud Dervilly-Pinel ${ }^{1}$. \\ Bruno Le Bizec ${ }^{1}$
}

\begin{abstract}
An untargeted strategy aiming at identifying non-intentionally added substances (NIAS) migrating from coatings was developed. This innovative approach was applied to two polyester-polyurethane lacquers, for which suppliers previously provided the identity of the monomers involved. Lacquers were extracted with acetonitrile and analyzed by liquid chromatography-high resolution mass spectrometry (LC-HRMS). Data, acquired in the full scan mode, were processed using an open-source Renvironment (xcms and CAMERA packages) to list the detected features and deconvolute them in groups related to individual compounds. The most intense groups, accounting for more than $85 \%$ of cumulated feature intensities, were then investigated. A homemade database, populated with predicted polyester oligomer combinations from a relevant selection of diols and diacids, enabled highlighting the presence of 14 and 17 cyclic predicted polyester oligomers in the two lacquers, including three mutual combinations explained by common known monomers. Combination hypotheses were strengthened by chromatographic considerations and by the investigation of fragmentation patterns. Regarding unpredicted migrating substances, four monomers were hypothesised to explain several polyester or caprolactam oligomer series. Finally, considering both predicted and tentatively elucidated unpredicted oligomers, it was possible to assign hypotheses to features representing up to $82 \%$ and $90 \%$ of the cumulated intensities in the two lacquers, plus $9 \%$ and $3 \%$ (respectively) originating from the procedural blank.
\end{abstract}

Keywords Food contact material · Non-intentionally added substance · Oligomer · Untargeted approach · HRMS screening · Chemical food safety

Ronan Cariou

laberca@oniris-nantes.fr

1 LABERCA, Oniris, INRA, Université Bretagne-Loire, Route de Gachet, 44307 Nantes, France

2 CEISAM, Université de Nantes, UMR CNRS 6230, 2 rue de la Houssinière, 44322 Nantes, France

3 ARDAGH MP WEST France SAS, La Ratière, 72200 Crosmières, France

\section{Introduction}

In order to preserve food quality, the inner surfaces of metallic food contact materials (FCMs) are coated with polymeric coatings acting as protective barriers. Recognized as the unsurpassed standard solution in terms of technical and economical performances, epoxy-resins based on bisphenol A (BPA) diglycidyl ether have traditionally and extensively been used worldwide. However, BPA was subjected to increasing pressure due to the consumers and scientific community concerns related to endocrine disruption effects [1] leading to regulatory restrictions at the EU level [2], including a complete ban for FCM applications in France [3, 4]. Polyester-based lacquers have grown as workable alternative coatings, but data gaps related to the chemical food safety need to be filled.

At the European Union level, FCMs are covered by the framework regulation EC 1935/2004 [5]. Article 3 states that 
under normal or foreseeable conditions of use, FCMs shall not transfer their constituents to food in quantities that could endanger human health. Regarding varnishes and coatings, no specific measures are in force. In practice, industrial stakeholders rely on a Partial Agreement guideline, AP (2004)1 [6], specifying a positive list of authorized monomers and additives. For migration testing, industrial stakeholders refer to the Commission Regulation 10/2011/EU [7] dedicated to plastic FCMs as a guideline. Interestingly, this regulation also defines non-intentionally added substances (NIAS) as impurities from starting materials, decomposition, or reaction products.

When dealing with polyester coatings, NIAS may result from incomplete polymerization reactions with solvents and additives, including reticulating agents and pigments or during the curing process. However, NIAS may also result from thermal or hydrolytic degradation over time. NIAS are commonly subdivided into two distinct categories, i.e., predictable and unpredictable compounds. Indeed, depending on the polyol and polyacid monomers formulation provided by lacquer suppliers, complex mixtures of linear, branched, and cyclic oligomers may thus be predicted. Any other compounds are considered as unpredicted NIAS that may derive from product compounds after reaction with solvents, additives, or impurities. For instance, in polyester-polyurethane lacquers, isophorone diisocyanate (IPDI) and caprolactam are commonly used as cross-linking and blocking agent, respectively [8], and may be involved in such unexpected reactions.

In order to objectivize NIAS occurrence and consequently enable a chemical risk assessment to be conducted, their characterization is required. NIAS substances are not characterized by any mass range and can either refer to very small compounds (few dozens of g.mol ${ }^{-1}$ ) to much larger ones, without any theoretical limit. Such a wide chemical diversity leads to analytical issues (range of physico-chemical properties exhibited, lack of analytical standards) impairing a proper risk characterization to be conducted [9-11]. For toxicokinetic investigations, NIAS exhibiting a molecular weight above $1000 \mathrm{Da}$ are not taken into consideration since they are unlikely to be absorbed through the gastrointestinal tract [12]. Moreover, a potential hydrolysis may be considered, especially of esters [12]. Although the literature reports on the characterization of NIAS migrating from polyethylene terephthalate used in bottle packaging [13-21], polybutylene terephthalate used for kitchen utensils [22], polycarbonate tableware [23], or biodegradable food packaging [24], only a limited number of studies are available for NIAS migrating from epoxy-phenolic [25, 26], polyvinyl chloride [27], polyester-polyphenolic [28], and polyester polyurethane can coatings [28-30]. Regarding polyester polyurethane oligomers, their separation using gas chromatography (GC) appears interesting to highlight volatile or non-polar NIAS such as those resulting from solvents or nitrogenous interactions. Liquid chromatography (LC), however. has proven its complementarity for compounds exhibiting molecular weights up to 1000 g.mol ${ }^{-1}$ [28-30]. For detection purposes at trace levels, mass spectrometry (MS) is considered as state of the art and has been implemented with low [28] (quadrupole) to high [29, 30] (time of flight, Orbitrap) resolution MS instruments. Besides the analytical challenge, NIAS identification is also an issue since neither corresponding databases nor pure analytical standards are available. To overcome such a challenge, high resolution MS strategies may be a good option for an increased confidence in peak assignments [31].

In the present work, an innovative approach aiming at identifying NIAS migrating from polyester-polyurethane coatings was proposed. In theory, NIAS migrating from lacquer can represent a multitude of compounds. To encompass such a possible diversity, a global untargeted analytical strategy was developed using liquid chromatography (LC) coupled to high resolution mass spectrometry (HRMS) to characterize lacquer extracts and give access to their fingerprints. Hence, specific data processing was needed to address the huge amount of data generated. In addition, to enable high throughput NIAS identification, the detected compounds were compared with a specifically developed homemade database based on their exact monoisotopic mass. This strategy allowed proposing an identification for the most intense migrating predicted and unpredicted compounds. Further, an in-depth investigation of the fragmentation patterns and chromatographic behaviors increased the level of confidence in identification. Such an innovative strategy is expected to increase knowledge of emerging chemical hazards and provide valuable information for efficient risk analysis.

\section{Material and methods}

\section{Chemicals}

Acetonitrile for sample preparation was provided by Carlo Erba Réactifs (HPLC grade, Rodano, Italy). Ultrapure water was purified using a Milli-Q-osmosis system from Millipore (Milford, MA, USA). Water and acetonitrile for mobile phase were obtained from Sigma-Aldrich (LC-MS ChromaSolv grade, St. Louis, MO, USA). Ammonium acetate salt (Emsure grade) was purchased from Merck (Darmstadt, Germany).

\section{Samples}

Two different polyester-polyurethane lacquers were obtained from two major industrial suppliers. According to the suppliers' information, both lacquers contained a mixture of terephthalic and isophthalic acid (PA) isomers as diacids. The diol monomers differed since lacquer 1 (Lac1) was based 
on neopentyl glycol (NPG) and 1,6-hexanediol (HD), while lacquer 2 (Lac2) was based on NPG, diethylene glycol (DEG), and ethylene glycol (EG). Both lacquers were coated on one side of metal plates and cured according to the supplier technical recommendations.

\section{Sample preparation}

The sample preparation strategy intended to be non-selective in order to favor the extraction of the largest number of compounds, thus covering a range of physico-chemical properties. Triplicate pieces $\left(0.5 \mathrm{dm}^{2}\right.$ each $)$ of coated plates were cut into $1 \mathrm{~cm}^{2}$ pieces and placed in $40 \mathrm{~mL}$ glass tubes. Extraction was performed using $10 \mathrm{~mL}$ of acetonitrile at $40^{\circ} \mathrm{C}$ during $24 \mathrm{~h}$. Aliquots of $2 \mathrm{~mL}$ were concentrated to $40 \mu \mathrm{L}$ at $40^{\circ} \mathrm{C}$ under a gentle stream of nitrogen. After addition of $160 \mu \mathrm{L}$ water, vortex mixing, and centrifugation, supernatants were transferred to new vials. Triplicate procedural blanks (without plate) were submitted to the same protocol. Glassware (tubes, vials, and Pasteur pipettes) was baked at $400^{\circ} \mathrm{C}$ for $4 \mathrm{~h}$ prior to use to minimize procedural contamination. Aluminium foil was used as barrier between tube caps and extracts.

\section{Instrumental acquisition}

An UltiMate 3000 UHPLC coupled to an Orbitrap Q-Exactive mass spectrometer fitted with a heated ESI source (HESI, ThermoFisher Scientific, San José, CA, USA) was used for data acquisition. External calibration was performed by infusing calibration mixture for negative (MSCAL6-1EA) and positive (MSCAL5-1EA) ionization mode (Supelco, Bellefonte, PA, USA). A volume of $10 \mu \mathrm{L}$ was injected on a reversedphase Hypersil Gold analytical column $(100 \mathrm{~mm} \times 2.1 \mathrm{~mm}$, $1.9 \mu \mathrm{m})$ (ThermoFisher Scientific) kept at $40{ }^{\circ} \mathrm{C}$. Mobile phases were composed of $10 \mathrm{mM}$ ammonium acetate in both water (A) and acetonitrile (B). The gradient began with (A/B) $80: 20(\mathrm{v} / \mathrm{v})$, ramped linearly to $0: 100$ over $16 \mathrm{~min}$, to be maintained for $2 \mathrm{~min}$ before returning in $2 \mathrm{~min}$ and stabilizing at initial conditions. The flow rate was set at $0.4 \mathrm{~mL} \cdot \mathrm{min}^{-1}$. In the positive mode, ionization parameters were as follows: sheath gas flow, 50 arbitrary units (AU); auxiliary gas flow, $10 \mathrm{AU}$; capillary temperature, $350{ }^{\circ} \mathrm{C}$; heater temperature, $300{ }^{\circ} \mathrm{C}$; spray voltage, $3.5 \mathrm{kV}$; S-lens radio frequency, $70 \mathrm{AU}$. In the negative mode (separate injections), the same parameters were used except for the spray voltage, which was set at $-2.5 \mathrm{kV}$. HRMS data were acquired in full scan mode over the mass-tocharge ratio $(\mathrm{m} / \mathrm{z})$ range $155-1200$ at a resolving power of 140,000 full width half maximum (FWHM) at $\mathrm{m} / \mathrm{z}, 200$. Automatic gain control (AGC Target) was set at high dynamic range $\left(5 \times 10^{5}\right)$ and maximum injection time (IT) at $500 \mathrm{~ms}$. Additionally, a selection of 22 precursor ions was fragmented in the positive targeted-MS ${ }^{2}$ mode using higher energy collisional dissociation (HCD) after unit mass quadrupole filtering, with parameters as follows: resolving power, 70,000; AGC Target, $1 \times 10^{5}$; maximum IT, $100 \mathrm{~ms}$; isolation window 0.4 uma, and normalized collision energy (NCE) from $10 \%$ to $35 \%$.

\section{Post-acquisition data analysis}

Extracting useful information from large full scan HRMS acquired data sets requires automatic processing, which was performed under the open source programming $\mathrm{R}$ environment. First, raw data (.raw) were converted to the open format . $m z X M L$ using the open access msConvert software (ProteoWizard). Second, in order to extract chromatographic features, the converted datasets were processed by the $x \mathrm{cms} \mathrm{R}$ package using the centWave peak detection algorithm [32], as previously described [33], with slight modifications. In these R-packages, a feature corresponds to a chromatographic peak from a given ion. Parameters were optimized for the present study for noise filtering and deconvolution to resolve coeluting ions: method, "centWave"; ppm, 5; prefilter, $\mathrm{c}(5,10000)$; snthresh, 6; peakwidth, $\mathrm{c}(1,15)$; mzdiff, 0.002; noise, 0 . A table report in .csv file format was then created for each data file ( $\mathrm{n}=3$ blanks, $\mathrm{n}=3$ Lac1, and $\mathrm{n}=3$ Lac2), where each feature was defined by a precise mass $(\mathrm{m} / \mathrm{z})$, a retention time $\left(-t_{R}, \min \right)$, and a signal intensity (area). Then, the nine lists of features were aligned according to the Retcor function ( $x \mathrm{cms}$ R-package), leading to a new table report in .$c s v$ file format where each feature was defined by common and precise $(\mathrm{m} / \mathrm{z})$ and $t_{\mathrm{R}}(\mathrm{min})$ as well as an individual signal intensity (area) for each sample [34]. Used parameters were: bw, 1; mzwid, 0.002; minfrac, 1; minsamp, 1. Usually, the insource ionization of one compound generates several ion species such as isotopologues, adducts, or fragments. Hence, the fourth and last step consisted of a deconvolution of the data table using the CAMERA R-package [35], which pairs signals potentially originating from a same compound (peak shape) in a so-called peak correlation group (PCgroup) and further suggests isotope and adduct assignments according to mass differences.

\section{Software}

Xcalibur Qual Browser software (ThermoFisher Scientific) was used to generate an elemental formula $(\mathrm{C}, \mathrm{H}, \mathrm{O}$, and $\mathrm{N}$ selected atoms) from a measured mass along with a degree of unsaturation (DoU) and a ppm deviation compared with the theoretical mass. ACD/MS Fragmenter software (Advanced Chemistry Development, ACD, Toronto, ON, Canada) was used to predict fragments from a hypothetic molecular structure draw. Suggested fragments were calculated based on established mass spectrometry fragmentation rules. The parameters selected were atmospheric pressure ionization technique, positive mode, and five fragmentation steps with the 
possibilities of all the common reactions (aromatic bonds cleavage, resonance reaction, rings formation, and hybrid shift). ChemSketch software (ACD) was used to estimate the $\log (\mathrm{P})$ values of the diol carbon chains.

\section{Homemade database of predicted oligomers}

A homemade database was developed and populated considering nine polyols (eight diols and one triol) and four polyacids (three diacids and one triacid) known to be potentially used as monomers in lacquer polyester formulations intended for food contact coatings, according to the literature [28-30] and guidelines [6, 7] (Table 1). All plausible monomer combinations up to dodecamers were enumerated, leading to more than 1 million combinations. Then, each combination was further enumerated in possible linear, partially, and fully cyclized forms (depending on the number of $\mathrm{H}_{2} \mathrm{O}$ losses), resulting in a higher number of oligomer structures. Finally, all combinations above 1000 Da were discarded, leading to 80,639 unique monomer and elemental formula combinations, involving up to 11 monomers. However, only 4682 distinct monoisotopic masses ranging from 172 to $998 \mathrm{Da}$ were obtained, due to various monomer combinations for a given elemental formula. More details about the database are available in the Electronic Supplementary Material (ESM, Figs. S1 and S2, Tables S1 and S2).

\section{Results and discussion}

Acetonitrile was selected as a strong extractive solvent to maximize extraction efficiency in order to facilitate the identification of substances possibly migrating in food or food simulants. Triplicate analyses of the three conditions (blank, Lac1, and Lac2) appeared to be highly repeatable. In the negative mode, both lacquers' total ion chromatograms (TICs) appeared similar to those of the procedural blanks (ESM Fig. S3a). Data insight revealed, however, some lacquerspecific signals but with a relatively low sensitivity. Such an observation was in accordance with previous findings reported by Paseiro-Cerrato and co-workers, who detected a few linear oligomers in the negative mode [30]. In the present work, when compared with the positive ionization mode observations, it was considered that the negative mode did not bring any significant additional information, since more intense related signals were usually observed in the positive mode. Similar conclusions were already reported by Schaefer and collaborators [28] and Bradley and co-workers [29], who selected the positive mode to track NIAS from polyester-polyurethane coatings. Consequently, no further investigations were conducted on the negative ion mode data. In the positive mode, Lac1 and Lac2 TICs were significantly different from the blank and from one another, certainly due to formulation differences (ESM Fig. S3b). The postacquisition data analysis workflow was then applied to the positive ion mode data set and the results were acutely reviewed, as described hereafter.

\section{Data processing}

Features eluting before $0.66 \mathrm{~min}$ and after $18 \mathrm{~min}$, which correspond to the dead volume and the end of the organic plateau, respectively, were discarded. PCgroups highlighted that major ion adducts were $\left[\mathrm{M}+\mathrm{NH}_{4}\right]^{+}$followed by $[\mathrm{M}+$ $\mathrm{H}]^{+},\left[\mathrm{M}+\mathrm{NH}_{4}+\mathrm{CH}_{3} \mathrm{CN}\right]^{+},[\mathrm{M}+\mathrm{Na}]^{+},[\mathrm{M}+\mathrm{K}]^{+}$, and $[\mathrm{M}+$ $\left.\mathrm{Na}+\mathrm{CH}_{3} \mathrm{CN}\right]^{+}$, generally at similar intensity ratios $(\mathrm{ESM}$ Table S3). Thus, considering a limit of $1000 \mathrm{Da}$ as upper mass limit [12] adjusted for the heaviest adduct $[\mathrm{M}+\mathrm{Na}+$ $\left.\mathrm{CH}_{3} \mathrm{CN}\right]^{+}$, features exhibiting $\mathrm{m} / \mathrm{z}$ above 1064.01632 were also discarded. Finally, considering the $0.66-18 \mathrm{~min} \mathrm{t}_{\mathrm{R}}$ and $155-1064 \mathrm{~m} / \mathrm{z}$ ranges in the positive mode, the peak picking process generated 26,519 features, which was decreased to 3977 features paired into 2293 PCgroups after alignment. Cumulated mean feature intensities reached $2.17 \times 10^{9}, 1.47$ $\times 10^{10}$, and $3.58 \times 10^{10}$ in blank, Lac1, and Lac2 triplicates. The 100 most intense PCgroups (1325 features) represented $85 \%$ (Lac1) and $91 \%$ (Lac2) of the cumulated mean feature intensities and were manually checked (ion chromatogram shapes, adducts nature, molecular mass suggestions, missing features, PCgroups merging). Among these most intense PCgroups (some being isomers), $33 \%$ and $50 \%$ were specific to Lac1 and Lac2, respectively, whereas $17 \%$ were common, attesting to expected similarities in these lacquers since NPG and PA were shared monomers. Interestingly, $3 \%$ out of the $17 \%$ common PCgroups were not related to lacquers since they were also observed in blank samples.

\section{Predicted oligomers}

\section{Homemade database search}

Among the most intense lacquer-specific PCgroups and as compiled in Table 2, $61 \%$ matched the homemade database ( $<2 \mathrm{ppm}$ deviation) as predicted combinations, based on known monomer formulations. Among these PCgroups, 28 and 27 were specific to Lac1 and Lac2, respectively, whereas six were mutual. PCgroups corresponding to isomers were gathered into predicted combinations, so that 11 and 14 predicted combinations were specific to Lac1 and Lac2, respectively, whereas three were common. Mass deviations were all found to be consistent at $<1.5 \mathrm{ppm}$, with a slight trend to decrease with an increasing $\mathrm{m} / \mathrm{z}$ ratio (ESM Fig. S4). As shown in Fig. 1, predicted combinations listed in Table 2 explained most peaks visible on the base peak chromatograms.

In terms of involved monomers, common predicted combinations were all and exclusively based on NPG and PA only, 
Table 1 Polyol and polyacid monomers selected to populate the homemade database. SML: specific migration limit (mg.kg $\left.{ }^{-1}\right)$; SML(T): total specific migration limit $\left(\mathrm{mg}_{\mathrm{kg}} \mathrm{g}^{-1}\right)$; N.i.: not included. *use reported by Paseiro-Cerrato and collaborators [30]

\begin{tabular}{|c|c|c|c|c|c|c|}
\hline Abbreviation & Name & Formula & Monoisotopic mass & Functions & AP $2004(6)$ & $\operatorname{Reg} 10 / 2011(7)$ \\
\hline EG & Ethylene glycol & $\mathrm{C}_{2} \mathrm{H}_{6} \mathrm{O}_{2}$ & 62.03678 & 2 & $\operatorname{SML}(T)=30$ & N.i. \\
\hline \multirow[t]{2}{*}{ PG } & 1,2-propanediol & \multirow[t]{2}{*}{$\mathrm{C}_{3} \mathrm{H}_{8} \mathrm{O}_{2}$} & \multirow[t]{2}{*}{76.05243} & \multirow[t]{2}{*}{2} & No SML & No SML \\
\hline & 1,3-propanediol & & & & $\mathrm{SML}=0.05$ & $\mathrm{SML}=0.05$ \\
\hline \multirow[t]{3}{*}{$\mathrm{BD}$} & 1,3-butanediol & \multirow[t]{3}{*}{$\mathrm{C}_{4} \mathrm{H}_{10} \mathrm{O}_{2}$} & \multirow[t]{3}{*}{90.06808} & \multirow[t]{3}{*}{2} & No SML & No SML \\
\hline & 1,4-butanediol & & & & $\operatorname{SML}(T)=5$ & No SML \\
\hline & 2-Methyl-1,3-propanediol & & & & $\mathrm{SML}=5$ & N.i. \\
\hline NPG & Neopentyl glycol & $\mathrm{C}_{5} \mathrm{H}_{12} \mathrm{O}_{2}$ & 104.08373 & 2 & $\mathrm{SML}=0.05$ & $\mathrm{SML}=0.05$ \\
\hline DEG & Diethylene glycol & $\mathrm{C}_{4} \mathrm{H}_{10} \mathrm{O}_{3}$ & 106.06300 & 2 & $\operatorname{SML}(\mathrm{T})=30$ & N.i. \\
\hline HD & 1,6-Hexanediol & $\mathrm{C}_{6} \mathrm{H}_{14} \mathrm{O}_{2}$ & 118.09938 & 2 & $\mathrm{SML}=0.05$ & $\mathrm{SML}=0.05$ \\
\hline HMP & 1,1,1-Tris(hydroxymethyl) propane & $\mathrm{C}_{6} \mathrm{H}_{14} \mathrm{O}_{3}$ & 134.09430 & 3 & $\mathrm{SML}=6$ & $\mathrm{SML}=6$ \\
\hline CHDM & 1,4-Cyclohexanedimethanol & $\mathrm{C}_{8} \mathrm{H}_{16} \mathrm{O}_{2}$ & 144.11503 & 2 & No SML & No SML \\
\hline TMP & 2,2,4-Trimethylpentane-1,3-diol & $\mathrm{C}_{8} \mathrm{H}_{18} \mathrm{O}_{2}$ & 146.13068 & 2 & To be fixed & N.i. \\
\hline AA & Adipic acid & $\mathrm{C}_{6} \mathrm{H}_{10} \mathrm{O}_{4}$ & 146.05791 & 2 & No SML & No SML \\
\hline \multirow[t]{3}{*}{ PA } & Orthophthalic acid & \multirow[t]{3}{*}{$\mathrm{C}_{8} \mathrm{H}_{6} \mathrm{O}_{4}$} & \multirow[t]{3}{*}{166.02661} & \multirow[t]{3}{*}{2} & No SML & No SML \\
\hline & Terephthalic acid & & & & $\mathrm{SML}=7.5$ & No SML \\
\hline & Isophthalic acid & & & & $\operatorname{SML}(\mathrm{T})=5$ & No SML \\
\hline NA* & Nadic acid & $\mathrm{C}_{9} \mathrm{H}_{10} \mathrm{O}_{4}$ & 182.05791 & 2 & N.i. & N.i. \\
\hline TMA & Trimellitic acid & $\mathrm{C}_{9} \mathrm{H}_{6} \mathrm{O}_{6}$ & 210.01644 & 3 & $\operatorname{SML}(T)=5$ & No SML \\
\hline
\end{tabular}

confirming expected similarities between both lacquers. Other predicted combinations involved at least HD and PA for Lac1 and DEG/EG and PA for Lac2. In terms of structure, predicted oligomer combinations were mainly cyclic tetramers, hexamers, and octamers, except for two trimers observed as linear in Lac1. These results are in line with those of Schaefer and co-workers [28] and Bradley and collaborators [29] who reported that cyclic oligomers were dominating linear oligomers, unlike those of PaseiroCerrato and co-workers [30], who reported the opposite. Considering that oligomers are formed during the ester polymerization (resin step), it is expected that linear oligomers would be mostly covalently bound to the tridimensional polyurethane network and/or would react with additives, whereas (unreactive) cyclic oligomers would be stable and subjected to migrate through the network. In terms of chain length, cumulated tetramer intensities were higher compared with those of hexamers, themselves higher compared with those of octamers. This trend can be explained by differences related to reactivity, migration, extraction solvent affinity, and/or ionization yield, so that no conclusion can be drawn in terms of relative concentrations. However, the relative intensity of predicted cyclic tetramer among all predicted cyclic oligomer combinations was higher in Lac2 (90\%) than in Lac1 (47\%) (ESM Fig. S5), suggesting that processes significantly influence the formation and/or the migration of oligomers according to the chain length.

\section{Chromatographic consistency}

Consistency of information deduced from the confrontation of measured exact masses and the database was further investigated in terms of chromatographic considerations.

First, it could be observed that for oligomers constituted of the same monomers, (for example No. 1, 2, and 3, Table 2), the retention time was increasing according to the chain length (see ESM Fig. S6 for illustrations). Indeed, cyclic tetramers were eluted first, followed by cyclic hexamers, then cyclic octamers, attesting to the decreasing polarity of these compounds, as previously observed by Bradley and co-workers [29]. Moreover, most of the time several peaks could be detected for a given combination because of the presence of terephthalic and isophthalic acid isomers in both lacquers as well as the possibilities of arrangements within the chain. Chromatographic separation was rather satisfactory for cyclic tetramers because of the limited number of isomers but hardly and not achievable for cyclic hexamers and octamers, respectively, due to the increasing number of isomers.

In terms of polarity and considering variations in diols composition, a consistent impact was observed. Indeed, using the molecular modelling ChemSketch software, $\log (\mathrm{P})$ values were estimated for each carbon chain diol monomer, i.e., without the alcohol functions, which were considered reacted as part of ester bounds to PA diacid monomers (e.g., ethane instead of $E G)$. Therefore, calculated $\log (\mathrm{P})$ values were found to be $0.98,1.82,3.05$, and 3.94 for DEG, EG, NPG, and HD 
Table 2 Most probable predicted oligomer combinations among the most intense PCgroups, according to database

\begin{tabular}{|c|c|c|c|c|c|c|c|c|c|}
\hline $\begin{array}{l}\text { Predicted } \\
\text { combination } \\
\mathrm{N}^{\mathrm{o}}\end{array}$ & $\begin{array}{l}\text { Mean measured } \\
{\left[\mathrm{M}+\mathrm{NH}_{4}\right]^{+} m / z}\end{array}$ & $\begin{array}{l}\mathrm{t}_{\mathrm{R}} \text { range } \\
(\mathrm{min})\end{array}$ & $\begin{array}{l}\text { Number of } \\
\text { PGgroups }\end{array}$ & $\begin{array}{l}\text { Lac1 } \\
\text { intensity } \\
\left(10^{6} \mathrm{AU}\right)\end{array}$ & $\begin{array}{l}\text { Lac2 } \\
\text { intensity } \\
\left(10^{6} \mathrm{AU}\right)\end{array}$ & $\begin{array}{l}\text { Oligomers } \\
\text { combination }\end{array}$ & $\begin{array}{l}\text { Number of } \\
\text { monomers }\end{array}$ & Structure & $\begin{array}{l}\text { Mean } \\
\text { deviation } \\
(\mathrm{ppm})\end{array}$ \\
\hline 1 & 486.21289 & $9.95-10.40$ & 2 & 1250 & 2500 & $2 \mathrm{NPG}+2 \mathrm{PA}$ & 4 & Cyclic & 1.3 \\
\hline 2 & 720.30192 & $12.80-13.50$ & 3 & 244 & 504 & $3 \mathrm{NPG}+3 \mathrm{PA}$ & 6 & Cyclic & 0.6 \\
\hline 3 & 954.39106 & $14.47-15.00$ & 1 & 42.6 & 119 & $4 \mathrm{NPG}+4 \mathrm{PA}$ & 8 & Cyclic & 0.4 \\
\hline 4 & 500.22849 & $11.00-11.80$ & 2 & 2940 & - & $\mathrm{NPG}+\mathrm{HD}+2 \mathrm{PA}$ & 4 & Cyclic & 1.2 \\
\hline 5 & 748.33313 & $11.90-14.65$ & 3 & 1390 & - & $\mathrm{NPG}+2 \mathrm{HD}+3 \mathrm{PA}$ & 6 & Cyclic & 0.5 \\
\hline 6 & 514.24422 & $12.05-12.50$ & 1 & 1090 & - & $2 \mathrm{HD}+2 \mathrm{PA}$ & 4 & Cyclic & 1.3 \\
\hline 7 & 996.43788 & $15.45-16.05$ & 4 & 1050 & - & NPG+3HD+4PA & 8 & Cyclic & 0.3 \\
\hline 8 & 982.42209 & $15.12-15.75$ & 4 & 982 & - & $2 \mathrm{NPG}+2 \mathrm{HD}+4 \mathrm{PA}$ & 8 & Cyclic & 0.1 \\
\hline 9 & 734.31758 & $13.45-14.10$ & 3 & 927 & - & 2NPG+HD+3PA & 6 & Cyclic & 0.7 \\
\hline 10 & 762.34875 & $14.35-15.12$ & 3 & 723 & - & $3 \mathrm{HD}+3 \mathrm{PA}$ & 6 & Cyclic & 0.5 \\
\hline 11 & 1010.45361 & $15.70-16.40$ & 3 & 369 & - & $4 \mathrm{HD}+4 \mathrm{PA}$ & 8 & Cyclic & 0.3 \\
\hline 12 & 968.40703 & $14.78-15.48$ & 3 & 343 & - & $3 \mathrm{NPG}+\mathrm{HD}+4 \mathrm{PA}$ & 8 & Cyclic & 0.7 \\
\hline 13 & 384.23855 & $6.40-6.70$ & 1 & 72.0 & - & $2 \mathrm{HD}+\mathrm{PA}$ & 3 & Linear & 1.3 \\
\hline 14 & 370.22284 & $6.10-6.40$ & 1 & 51.7 & - & $\mathrm{NPG}+\mathrm{HD}+\mathrm{PA}$ & 3 & Linear & 1.2 \\
\hline 15 & 488.19216 & $8.16-9.00$ & 3 & - & 11,000 & $\mathrm{DEG}+\mathrm{NPG}+2 \mathrm{PA}$ & 4 & Cyclic & 1.3 \\
\hline 16 & 490.17139 & $5.67-7.48$ & 4 & - & 5770 & $2 \mathrm{DEG}+2 \mathrm{PA}$ & 4 & Cyclic & 1.3 \\
\hline 17 & 446.14514 & $6.95-7.60$ & 2 & - & 3140 & $\mathrm{EG}+\mathrm{DEG}+2 \mathrm{PA}$ & 4 & Cyclic & 1.3 \\
\hline 18 & 678.25511 & $11.65-12.30$ & 2 & - & 494 & $\mathrm{EG}+2 \mathrm{NPG}+3 \mathrm{PA}$ & 6 & Cyclic & 0.9 \\
\hline 19 & 722.28122 & $11.35-12.05$ & 3 & - & 370 & $\mathrm{DEG}+2 \mathrm{NPG}+3 \mathrm{PA}$ & 6 & Cyclic & 0.7 \\
\hline 20 & 680.23438 & $9.91-10.82$ & 2 & - & 286 & $\mathrm{EG}+\mathrm{DEG}+\mathrm{NPG}+3 \mathrm{PA}$ & 6 & Cyclic & 0.9 \\
\hline 21 & 724.26048 & $9.75-10.57$ & 1 & - & 155 & $2 \mathrm{DEG}+\mathrm{NPG}+3 \mathrm{PA}$ & 6 & Cyclic & 0.7 \\
\hline 22 & 956.37058 & $13.20-14.10$ & 1 & - & 118 & $\mathrm{DEG}+3 \mathrm{NPG}+4 \mathrm{PA}$ & 8 & Cyclic & 0.7 \\
\hline 23 & 912.34420 & $13.40-14.25$ & 2 & - & 87.1 & $\mathrm{EG}+3 \mathrm{NPG}+4 \mathrm{PA}$ & 8 & Cyclic & 0.5 \\
\hline 24 & 682.21360 & $8.29-8.88$ & 1 & - & 82.0 & $\mathrm{EG}+2 \mathrm{DEG}+3 \mathrm{PA}$ & 6 & Cyclic & 0.8 \\
\hline 25 & 914.32351 & $12.15-13.10$ & 2 & - & 72.5 & $\mathrm{EG}+\mathrm{DEG}+2 \mathrm{NPG}+4 \mathrm{PA}$ & 8 & Cyclic & 0.6 \\
\hline 26 & 444.16589 & $8.31-10.00$ & 1 & - & 72.0 & $\mathrm{EG}+\mathrm{NPG}+2 \mathrm{PA}$ & 4 & Cyclic & 1.3 \\
\hline 27 & 636.20833 & $10.29-11.10$ & 2 & - & 61.6 & $2 \mathrm{EG}+\mathrm{NPG}+3 \mathrm{PA}$ & 6 & Cyclic & 1.2 \\
\hline 28 & 870.29726 & $12.32-13.31$ & 1 & - & 26.0 & $2 \mathrm{EG}+2 \mathrm{NPG}+4 \mathrm{PA}$ & 8 & Cyclic & 0.6 \\
\hline
\end{tabular}

equivalents, respectively. Thus, the observed elution orders were in line with the predicted $\log (\mathrm{P})$ values, as illustrated in Fig. 2.

In brief, chromatographic considerations strengthened the identification assignments of predicted combinations to PCgroups.

\section{$M S^{2}$ fragmentation}

Beyond the exact mass determination leading to a chemical formula fitting a predicted oligomer and the consistency of chromatographic observations, it was advisable to increase the confidence level relative to the identification hypotheses. A selection of 15 predicted oligomer ions (when several isomers, the most intense) were subjected to fragmentation in the $\mathrm{MS}^{2}$ mode. These ions were representative in terms of nature of monomers, structure (cyclic and linear), and cycle length. Each oligomer ion was fragmented as $[\mathrm{M}+\mathrm{H}]^{+}$or $[\mathrm{M}+$ $\left.\mathrm{NH}_{4}\right]^{+}$at three different NCE by steps of $5 \%$ or $10 \%$ in order to generate a maximum of three simultaneous events. Each interpretation was carried out with the NCE (\%) allowing for the best compromise between fragments (intensity and information) and remaining signal of the precursor ion. In order to simplify the interpretation processing, only fragments detected above a relative intensity to the base peak of $5 \%$ were considered. The ACD/MS Fragmenter software was helpful to elucidate the structure of the main fragments (ESM Table S4). Indeed, fragments were often matching combinations of diol and diacid monomer present in the oligomer, with rearrangements after a loss of one molecule of water for example. In addition, and logically, the more the oligomer is composed of various monomers, the more the list of observed 


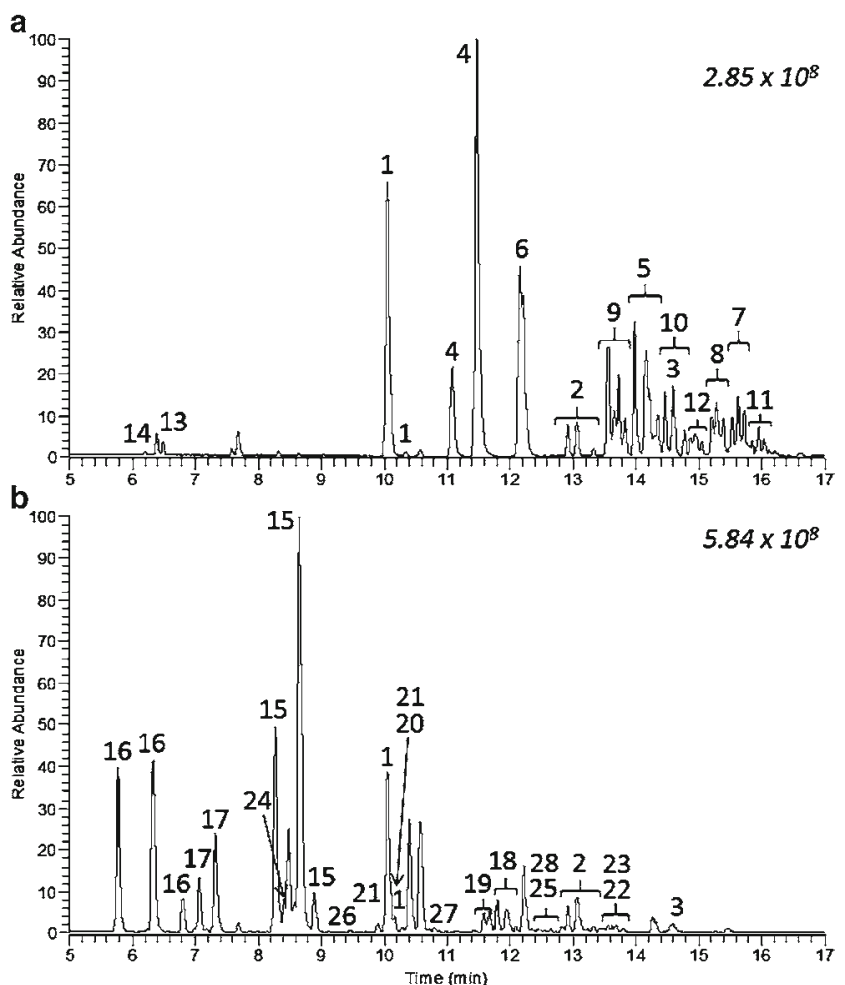

Fig. 1 Base peak chromatograms obtained for Lac1 (a) and Lac2 (b), along with predicted combination numbering from Table 2

fragments is long. Likewise, some fragment ions were specific to given monomers, such as at $\mathrm{m} / \mathrm{z}, 149$ and sometimes 167 for PA, $\mathrm{m} / \mathrm{z}, 69$ for NPG or $\mathrm{m} / \mathrm{z} 83$ for HD, respectively. Conversely, there was no specific fragment ion discriminating EG from DEG. Indeed, DEG is composed in some way of two EG and a rupture can occur on each side of the central oxygen, leading to EG-like fragments. The fragment ion at $\mathrm{m} / \mathrm{z} 193$ was intense and systematically detected in presence of PA with DEG and/or EG (ESM Table S4). Furthermore, even without a specific ion, the complete set of detected ions was sufficient to be confident concerning the identification. As a conclusion regarding predicted oligomers, a total of 28 monomer combinations could be elucidated, the collection of clues (known constituting monomers, precise $\mathrm{m} / \mathrm{z}$ matching database, retention time consistency, fragmentation pattern) was considered strong enough to reach the second level of identification as proposed by Sumner et al. in a context of biomarkers identification in untargeted (metabolomics) studies [36]. A higher level of confidence will require genuine analytical standards that are still not, commercially or not, available.

\section{Unpredicted compounds}

\section{Deduction of unexpected monomers}

Among the most intense lacquer-specific PCgroups, $36 \%$ did not match the database. Assuming that the presence of an

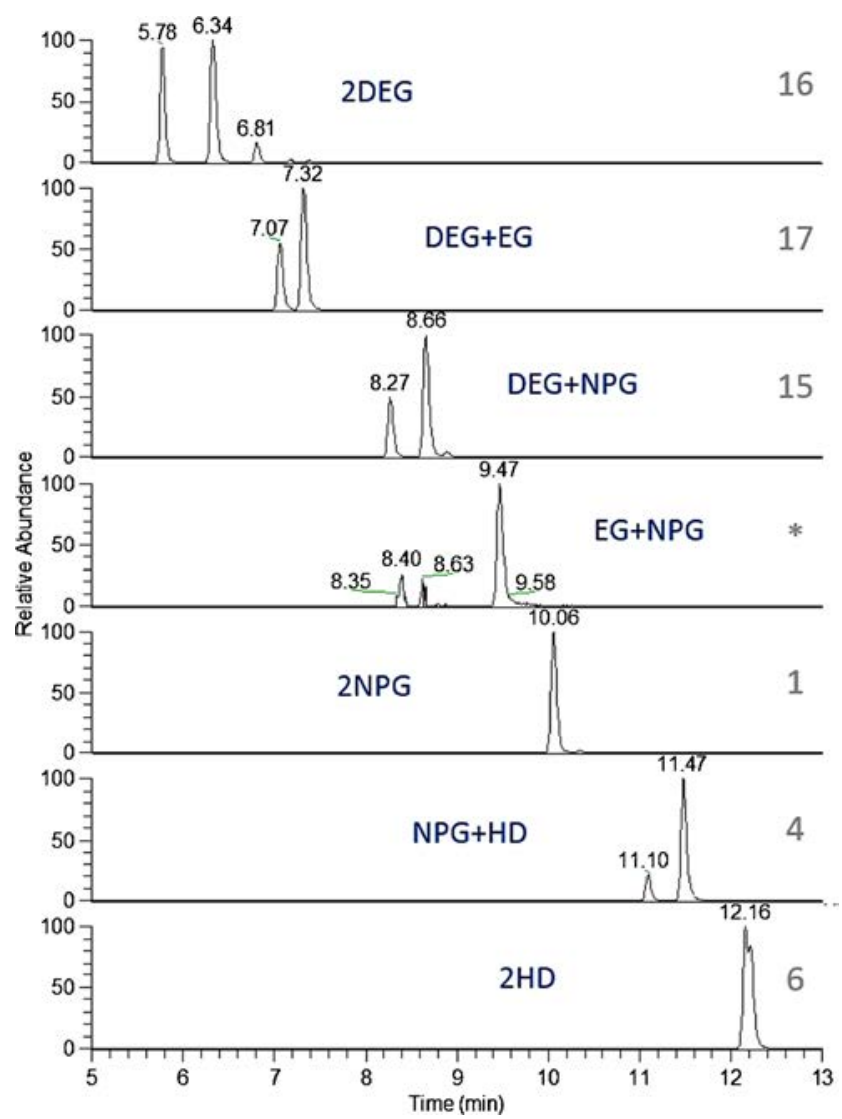

Fig. 2 Extracted ion chromatograms $( \pm 2 \mathrm{ppm})$ of predicted cyclic tetramers from Lac1 $(16,17,15, *$ and 1) and Lac2 (4 and 6). Acid monomers: PA only; numbering corresponds to predicted combinations provided in Table 2; *: not among the most intense PCgroups

unexpected starting monomer would lead to oligomer series, exact mass differences corresponding to the substitution of a known monomer by an unknown one were examined to reconstitute such series.

This way, 15 combinations $(\mathrm{C}, \mathrm{H}, \mathrm{O})$ involving 18 PCgroups were connected in several networks after retention time consistency verified as described above. Taking into account permuted monomers, $\mathrm{C}_{\mathrm{x}} \mathrm{H}_{\mathrm{y}} \mathrm{O}_{\mathrm{z}}$ elemental formula was assigned ( $<2 \mathrm{ppm}$ deviation) (Table 3 ). Considering results from predicted oligomers and observed intensities, cyclic structures appeared more plausible than linear ones. The lengths of the cyclic oligomers were estimated from the number of oxygen atoms. Indeed, cyclic tetramer, hexamer, and octamers can be composed of at least 8,12 , and 16 oxygen atoms, originating from the 4,6 , and 8 ester functions. Moreover, a number of PA monomers was hypothesized from the DoU. Indeed, the oligomer cycle along with the ester functions contribute to DoU of 5, 7, and 9 for cyclic tetramers, hexamers, and octamers, respectively, whereas a PA monomer bears an additional DoU of 4. Combining all this information, three distinct series were related to three tentative unexpected monomer formulas, including one diol $\left(\mathrm{C}_{12} \mathrm{H}_{24} \mathrm{O}_{2}\right)$ in Lac1, 


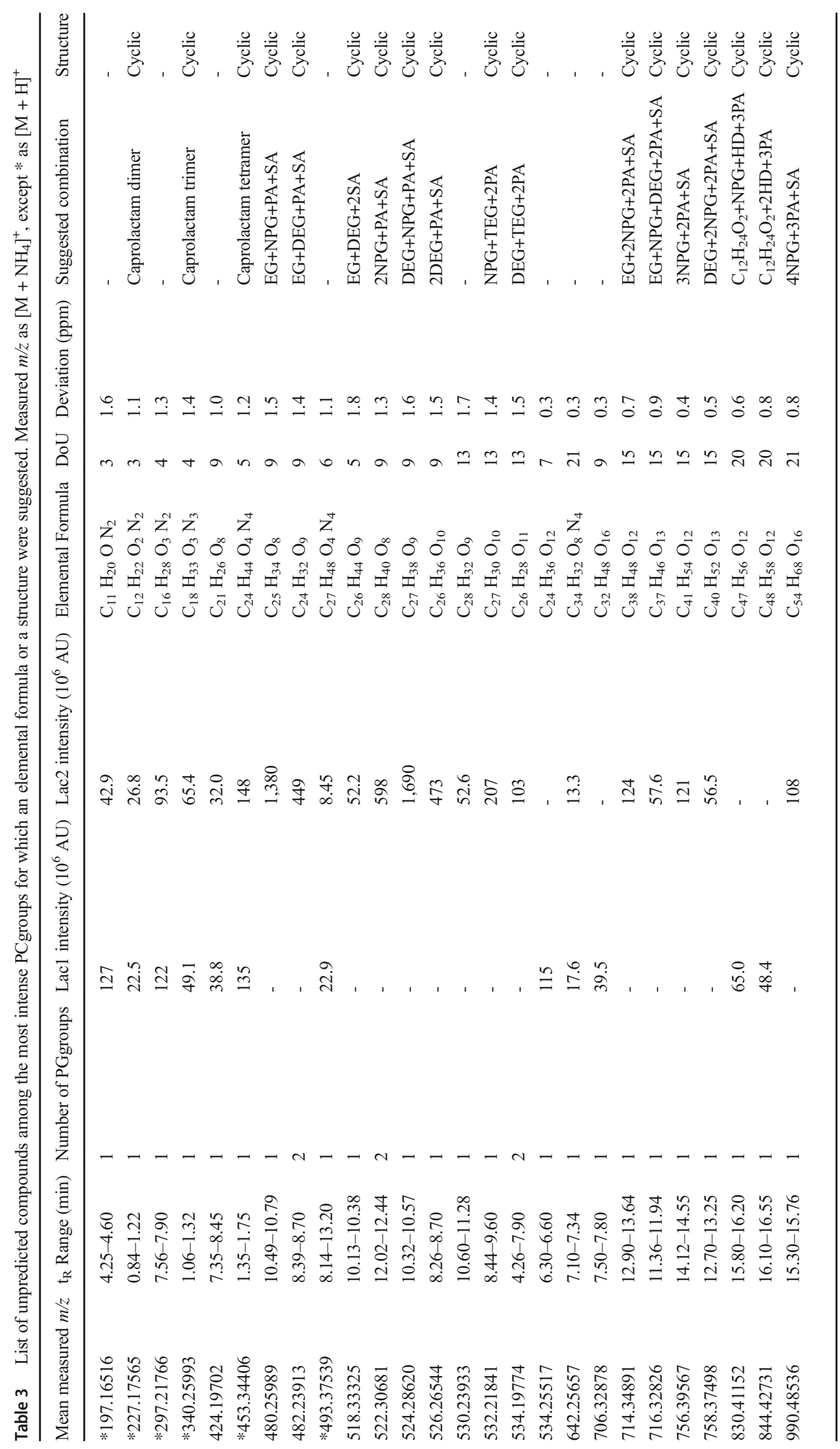


one diol $\left(\mathrm{C}_{6} \mathrm{H}_{14} \mathrm{O}_{4}\right)$ and one diacid $\left(\mathrm{C}_{10} \mathrm{H}_{18} \mathrm{O}_{4}\right)$ in Lac2 (Table 3). Regarding Lac2, suggestions of structures were attempted. The sebacic acid (SA), not initially included in the homemade database and even less considered as a known starting monomer since not subjected to restriction, was found as the only diacid matching the elemental formula in the AP (2004) 1 guideline [6]. Regarding the second diol, triethylene gycol (TEG) was suggested as a possible impurity of DEG and/or EG, due to relatively low intensities, even though it is also allowed without restriction in the AP (2004) 1 [6]. By contrast, regarding Lac1, no diol with the $\mathrm{C}_{12} \mathrm{H}_{24} \mathrm{O}_{2}$ elemental formula was found in this latter resolution, so that no structure could be suggested. Three tetramer combinations slightly less intense involving this diol and not present among the investigated PCgroups were detected in Lac1 (ESM Fig. S7).

Additionally, a series of nitrogenous compounds detected in Lac1 and Lac2 extracts were also observed as correlated. In this series, unlike for polyester oligomers, $[\mathrm{M}+\mathrm{H}]^{+}$forms were most intense ions, which may be explained by the presence of nitrogen. Moreover, low retention times associated indicate high polarity. The $\mathrm{C}_{6} \mathrm{H}_{11} \mathrm{ON}$ series pattern exhibits one DoU and corresponds exactly to caprolactam, which was known to be used as a blocking agent in both studied formula. Based on a study dealing with identification of caprolactam oligomers in nylon [37], relevant structures could be suggested in the present work. Indeed, compounds corresponding to $\mathrm{C}_{12} \mathrm{H}_{22} \mathrm{O}_{2} \mathrm{~N}_{2}, \mathrm{C}_{18} \mathrm{H}_{33} \mathrm{O}_{3} \mathrm{~N}_{3}$, and $\mathrm{C}_{24} \mathrm{H}_{44} \mathrm{O}_{4} \mathrm{~N}_{4}$ were assigned to caprolactam cyclic dimer, trimer, and tetramer, respectively (ESM Fig. S8). The caprolactam cyclic pentamer, hexamer, and heptamer were also detected in both lacquers but with lower intensities. In addition, the same trend was observed in Lac1 and Lac2 in terms of intensities of caprolactam cyclic oligomers (ESM Fig. S9). In line with the results of Jenke co-workers [37], retention times increased with the cycle size, which supports these hypotheses. Another study dealing with the identification of non-volatile migrants coming from multilayer materials revealed the migration of four caprolactam oligomers (dimer to pentamer), explained by the use of a polyamide layer [38]. However, layers were joined by polyurethane, which could also explain their presence. Moreover, a complementary analysis with $\mathrm{m} / \mathrm{z}$ range starting at 100 allowed detecting the elution of caprolactam itself between the dimer and the trimer as was observed by Jenke and collaborators (37), with intensities twice as intense as the tetramers.

\section{$\mathrm{MS}^{2}$ fragmentation}

With the same purpose as for predicted oligomers, four putative polyester cyclic tetramer ions $(\mathrm{TEG}+\mathrm{NPG}+2 \mathrm{PA}, \mathrm{NPG}+$ $\mathrm{DEG}+\mathrm{PA}+\mathrm{SA}, \mathrm{C}_{12} \mathrm{H}_{24} \mathrm{O}_{2}+\mathrm{NPG}+\mathrm{HD}+3 \mathrm{PA}$, and $\mathrm{C}_{12} \mathrm{H}_{24} \mathrm{O}_{2}+$ $2 \mathrm{HD}+3 \mathrm{PA}$ ) were fragmented in order to strengthen the hypothesis of unexpected monomers and suggest a structure for the $\mathrm{C}_{12} \mathrm{H}_{24} \mathrm{O}_{2}$ monomer. Regarding TEG combination (ESM Table S5a), similar fragments $(193,261,341$, and $427)$ were also detected after fragmentation for similar predicted combinations $(\mathrm{DEG}+\mathrm{NPG}+2 \mathrm{PA}, \mathrm{EG}+\mathrm{NPG}+2 \mathrm{PA}$ ) (ESM Tables S4k and S4m). These results were expected since TEG, DEG, and EG monomers belong to glycol-based series. Regarding the SA combination, specific fragments involving $\left[\mathrm{C}_{10} \mathrm{H}_{18} \mathrm{O}_{4}+\mathrm{H}\right]^{+}$, supposedly $[\mathrm{SA}+\mathrm{H}]^{+}$at $\mathrm{m} / z$ 203, were observed (ESM Table S5b). The two combinations involving the unexpected $\mathrm{C}_{12} \mathrm{H}_{24} \mathrm{O}_{2}$ monomer were also fragmented. Fragmentation patterns corroborated the presence of NPG, HD, and PA in the first combination (ESM Table S5c) and HD and PA in the second one (ESM Table S5d). However, the observed fragments could not help to propose a structure for the unknown diol. In the same way as for TEG, the low intensity exhibited by these two combinations suggests that the monomer may be an impurity.

Additionally, nitrogenous compounds were fragmented as well. Obtained patterns were similar to those reported by Jenke and collaborators [37], with the systematic detection of a fragment corresponding to the caprolactam monomer as base peak. Moreover, fragments corresponding to intermediate oligomers were also detected (ESM Table S6).

Although such a fragmentation strategy cannot be considered sufficient to confirm the composition of unpredicted NIAS, it has to be considered as a complementary piece of evidence in the identification process.

\section{Remaining features}

Among the remaining unpredicted compounds $(36 \%$ of the most intense lacquer-specific PCgroups), no elemental formula could be suggested after the PCgroup creation for seven of them. Eight others were assigned a tentative elemental formula (Table 3), including several nitrogencontaining compounds, in both lacquers. Such substances may arise from reactions between caprolactam and/or IPDI with monomers and/or solvents, but were not reviewed in the present work.

\section{Overview}

\section{Data processing completion}

In order to deal with the tremendous amount of information to be processed, it was decided to review PCgroups in the decreasing order of intensity, assuming that concentrations would follow the same trend. Although computing automation was helpful, gathering/sorting clues and checking consistency of inferences remained time-consuming for the operator. In the present work, the most intense PCgroups representing $85 \%-91 \%$ of the cumulative features intensities were reviewed. Table 4 summarizes the processing completion 
Table 4

Overview of data processing completion

\begin{tabular}{lll}
\hline & Lac1 & Lac2 \\
\hline Number of features & 4,431 & 5,405 \\
Cumulated intensity & $1.47 \times 10^{10}$ & $3.58 \times 10^{10}$ \\
Contribution of most intense PCgroups $(n)$ & $85 \%$ & $91 \%$ \\
Predicted ( $\mathrm{n}=61)$ & $79 \%$ & $70 \%$ \\
Tentatively elucidated $(\mathrm{n}=21)$ & $3.3 \%$ & $20 \%$ \\
Unpredicted ( $=15)$ & $2.8 \%$ & $1.0 \%$ \\
Blank (n = 3) & $0.10 \%$ & $0.06 \%$ \\
Non-reviewed features & $15 \%$ & $9.7 \%$ \\
Present in blank & $9.1 \%$ & $3.2 \%$ \\
Lacquer-specific & $6.0 \%$ & $6.5 \%$ \\
\hline
\end{tabular}

according to the reached conclusion for each PCgroup, expressed in terms of cumulative intensity. Total intensity was two times higher in Lac2 compared with Lac1, possibly due to the number and thickness of coating layers first, the amount being slightly more important for Lac2, but also the ability of each polymerization process to generate oligomers and let them migrate through the polymer network.

Within the considered $t_{R}$ range, blank contributions remained quite low, at $9.1 \%$ and $3.2 \%$ of the total feature intensity in Lac1 and Lac2, respectively, attesting to proper sample preparation and appropriate concentration to enhance lacquer-specific signals. Predicted oligomers, defined as compounds matching the database of expectable combinations of known monomers, accounted for the most important part, $79 \%$ and $70 \%$ of total feature intensities in Lac1 and Lac2, respectively. This substantial proportion gives evidence that most NIAS accessible with the proposed sample preparation and data acquisition, including LC and ionization techniques, are predicted cyclic oligomers. Three unexpected monomers and nitrogenous compounds were hypothesized, leading to tentatively elucidated cyclic oligomers representing up to $20 \%$ of the total feature intensity in Lac2, mainly due to the presence of an intended but unknown monomer (SA). Lastly, remaining unprocessed features, representing about $67 \%$ of the total features, contributed to only $15 \%$ and $9.7 \%$ of the total feature intensities in Lac1 and Lac2, respectively. These features could match the database but this raises the question of the relevance of reviewing such relatively low contributors without further indication on their actual concentrations.

\section{Estimated monomer contribution}

The information relative to the exact formulation of resins and lacquers, including ingredient proportions, is understandably kept confidential by the suppliers. However, we attempted to roughly estimate the monomer proportions considering their contributions to predicted and tentatively elucidated oligomer patterns. Indeed, for each monomer, cyclic polyester oligomer intensities were weighted relative to the chain length and summed, leading to a breakdown of intensities on a molar basis. This approximation does not take into account differential behaviors due to specific relativities, molar masses, migration, and ionisation rates. Results are shown in Fig. 3. As a mathematical consequence of the calculation equation, cumulative proportions of diols and diacids reach $50 \%$ in both lacquers, which is convenient since stoichiometric proportions would be expected. More interestingly, Lac1 seems to be composed of equivalent NPG and HD proportions, whereas Lac2 appears to be more complex. Indeed, regarding diols, DEG and NPG seem to be in similar proportions followed by EG at a lower rate, and PA seems to dominate the diacids part. Relatively low contributions of $\mathrm{C}_{12} \mathrm{H}_{24} \mathrm{O}_{2}$ diol to Lac1 and TEG to Lac2 suggest that these monomers are impurities rather than IAS.

\section{Conclusions and perspectives}

The proposed methodology allowed seeking, within complex LC-HRMS datasets, predicted oligomers as well as deducing unpredicted NIAS migrating from polyester-polyurethane coatings. Relying on open-source software, the data processing was automated, facilitating the manual review along with a homemade database dedicated to predicted combinations of known monomers. In the two lacquer samples investigated, predicted cyclic oligomers accounted for two-thirds to threequarters of the cumulated signal intensities. The remaining signals were attributed to elucidated structures based on unexpected monomers explaining consistent signal series, as well as procedural blank contribution and minor nonreviewed compounds. In terms of identification confidence, all suggestions were supported by chromatographic and fragmentation consistency.

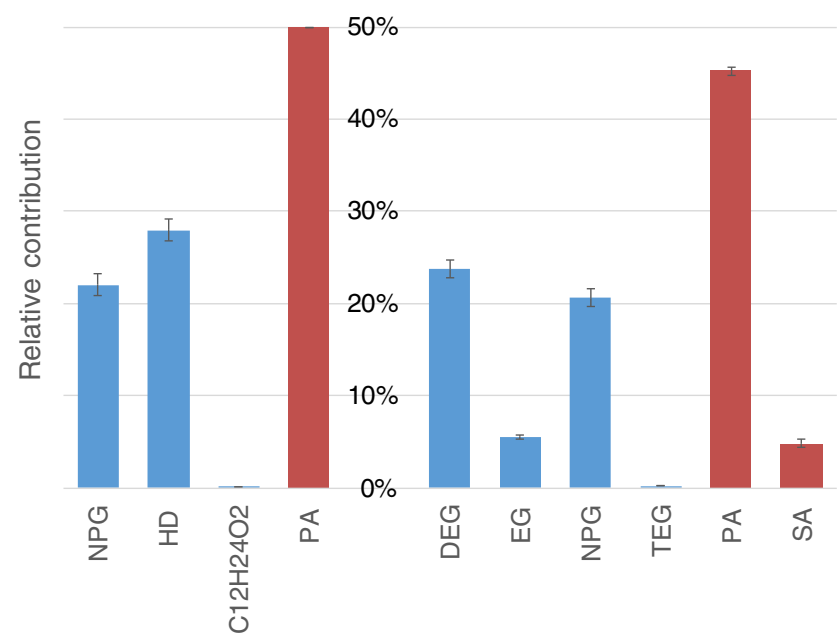

Fig. 3 Estimation of monomer relative contributions $(n=3)$ in Lac1 (left) and Lac2 (right) for cyclic oligomers 
The analytical strategy (LC-ESI-HRMS) proposed was well suited to investigate cyclic oligomers. For future identification purposes, the database will be updated in light of the outcomes of the present work, including in particular the three unexpected monomers, caprolactam oligomers, as well as combinations of monomers resulting from reaction with solvents or additives (IPDI, caprolactam). If cyclic oligomers appear as major migrating NIAS from polyesterpolyurethane coating, only quantification will allow to balance with linear oligomers or other NIAS, pending complementary analyses covering more volatile fractions by GC couplings, or to balance with migrating fraction above $1000 \mathrm{Da}$. Beyond identification, which was the purpose of the present study, future work should address the quantification of such migrating substances in food simulant as well as in foodstuffs in an exposure assessment perspective. Risk characterization represents another major data gap to be filled. Beforehand, a wide range of genuine analytical oligomer standards should be made available to the community.

Acknowledgments The authors are grateful to the two lacquer suppliers, Metlac (Bosco Marengo, Italy) and Valspar Corporation (Chipping Norton, UK), for the information provided on formulations.

\section{Compliance with ethical standards}

Conflict of interest The authors declare that there are no conflicts of interest.

\section{References}

1. European Chemical Agency. MSC unanimously agrees that Bisphenol $\mathrm{A}$ is an endocrine disruptor. ECHA/PR/17/12. [Internet]. 2017 [cited 2017 Jun 21]. Available from: https://echa. europa.eu/-/msc-unanimously-agrees-that-bisphenol-a-is-anendocrine-disruptor

2. Commission Implementing Regulation (EU) No 321/2011 of 1 April 2011 amending Regulation (EU) No 10/2011 as regards the restriction of use of Bisphenol A in plastic infant feeding bottles. Off J Eur Union. 2011;L 87/1.

3. LOI no 2010-729 du 30 juin 2010 tendant à suspendre la commercialisation de biberons produits à base de bisphénol A. J Off la République Française. 2010.

4. LOI no 2012-1442 du 24 décembre 2012 visant à la suspension de la fabrication, de l'importation, de l'exportation et de la mise sur le marché de tout conditionnement à vocation alimentaire contenant du bisphénol A. J Off la République Française. 2012.

5. European Commission. Regulation (EC) No 1935/2004 on materials and articles intended to come into contact with food and repealing Directives 80/590/EEC and 89/109/EEC. Off J Eur Union. 2004;L338/4.

6. Council of Europe Framework Resolution ResAP (2004) 1 on coatings intended to come into contact with foodstuffs. 2009.

7. European Commission. Regulation (EU) No 10/2011 on plastic materials and articles intended to come into contact with food. Off J Eur Union. 2011;L 12/1.

8. Wicks ZW. Blocked isocyanates. Prog Org Coatings. 1975;3(1): 73-99.
9. Nerin C, Alfaro P, Aznar M, Domeño C. The challenge of identifying non-intentionally added substances from food packaging materials: A review. Anal Chim Acta. 2013;775:14-24.

10. ILSI Europe. Guidance on best practices on the risk assessment of non intentionally added substances (NIAS) in food contact materials and articles. [cited 2017 Apr 27]; Available from: http://ilsi. org/wp-content/uploads/2016/05/2015-NIAS_version-January2016.pdf

11. Hoppe M, de Voogt P, Franz R. Identification and quantification of oligomers as potential migrants in plastics food contact materials with a focus in polycondensates - A review. Trends Food Sci Technol. 2016;50:118-30.

12. European Food Safety Authority. Food contact materials: Note for guidance for petitioners presenting an application for the safety assessment of a substance to be used in food contact materials prior to its authorisation. EFSA J. 2008;7:1-125.

13. Besnoin J-M, Choi KY. Identification and characterization of reaction byproducts in the polymerization of polyethylene terephthalate. J Macromol Sci Part C Polym Rev. 1989 Feb;29(1):55-81.

14. Milon H. Identification of poly(ethylene terephthalate) cyclic oligomers by liquid chromatography-mass spectrometry. J Chromatogr. 1991;554(1-2):305-9.

15. Barnes KA, Damant AP, Startin JR, Castle L. Qualitative liquid chromatographic-atmospheric-pressure chemical-ionisation mass spectrometric analysis of polyethylene terephthalate oligomers. J Chromatogr A. 1995;712(1):191-9.

16. Bryant JJL, Semlyen JA. Cyclic polyesters: 6. Preparation and characterization of two series of cyclic oligomers from solution ringchain reactions of poly(ethylene terephthalate). Polymer. 1997;38(10):2475-82.

17. Harrison AG, Taylor MJ, Scrivens JH, Yates H. Analysis of cyclic oligomers of poly(ethylene terephthalate) by liquid chromatography/mass spectrometry. Polymer. 1997;38(10):254955 .

18. Lim B, Kwon S, Kang E, Park H, Lee H, Kim W. Isolation and identification of cyclic oligomers of the poly(ethylene terephthalate)-poly(ethylene isophthalate) copolymer. J Polym Sci Part A Polym Chem. 2003;41(7):881-9.

19. Nasser ALM, Lopes LMX, Eberlin MN, Monteiro M. Identification of oligomers in polyethyleneterephthalate bottles for mineral water and fruit juice: Development and validation of a high-performance liquid chromatographic method for the determination of first series cyclic trimer. J Chromatogr A. 2005;1097(1):130-7.

20. Kim D-J, Lee K-T. Determination of monomers and oligomers in polyethylene terephthalate trays and bottles for food use by using high performance liquid chromatography-electrospray ionizationmass spectrometry. Polym Test. 2012;31(3):490-9.

21. Bignardi C, Cavazza A, Corradini C, Salvadeo P. Targeted and untargeted data-dependent experiments for characterization of polycarbonate food-contact plastics by ultra high performance chromatography coupled to quadrupole orbitrap tandem mass spectrometry. J Chromatogr A. 2014;1372:133-44.

22. Brenz F, Linke S, Simat T. Linear and cyclic oligomers in polybutylene terephthalate for food contact materials. Food Addit Contam Part A. 2017.

23. Bignardi C, Cavazza A, Laganà $\mathrm{C}$, Salvadeo $\mathrm{P}$, Corradini $\mathrm{C}$. Release of non-intentionally added substances (NIAS) from food contact polycarbonate: Effect of ageing. Food Control. 2017;71: 329-35.

24. Canellas E, Vera P, Nerín C. UPLC-ESI-Q-TOF-MS(E) and GCMS identification and quantification of non-intentionally added substances coming from biodegradable food packaging. Anal Bioanal Chem. 2015;407(22):6781-90.

25. Biedermann M, Grob K. Food contamination from epoxy resins and organosols used as can coatings: Analysis by gradient NPLC. Food Addit Contam. 1998 Jul 10;15(5):609-18. 
26. Bradley EL, Driffield M, Harmer N, Oldring PKT, Castle L. Identification of Potential Migrants in Epoxy Phenolic Can Coatings. Int J Polym Anal Charact. 2008;13(3):200-23.

27. Vaclavikova M, Paseiro-Cerrato R, Vaclavik L, Noonan GO, DeVries J, Begley TH. Target and non-target analysis of migrants from PVC-coated cans using UHPLC-Q-Orbitrap MS: evaluation of long-term migration testing. Food Addit Contam Part A. 2016;11:1-12.

28. Schaefer A, Ohm VA, Simat TJ. Migration from can coatings: Part 2. Identification and quantification of migrating cyclic oligoesters below 1000 Da. Food Addit Contam. 2004;21(4):377-89.

29. Bradley E, Driffield M, Guthrie J, Harmer N, Thomas Oldring PK, Castle L. Analytical approaches to identify potential migrants in polyester-polyurethane can coatings. Food Addit Contam Part A. 2009;26(12):1602-10.

30. Paseiro-Cerrato R, MacMahon S, Ridge CD, Noonan GO, Begley TH. Identification of unknown compounds from polyester cans coatings that may potentially migrate into food or food simulants. J Chromatogr A. 2016;1444:106-13.

31. Martínez-Bueno MJ, Hernando MD, Uclés S, Rajski L, Cimmino S, Fernández-Alba AR. Identification of non-intentionally added substances in food packaging nano films by gas and liquid chromatography coupled to orbitrap mass spectrometry. Talanta. 2017 Sep;172:68-77.

32. Tautenhahn R, Böttcher C, Neumann S. Highly sensitive feature detection for high resolution LC/MS. BMC Bioinformatics. 2008;9:504.

33. Cariou R, Omer E, Léon A, Dervilly-Pinel G, Le Bizec B. Screening halogenated environmental contaminants in biota based on isotopic pattern and mass defect provided by high resolution mass spectrometry profiling. Anal Chim Acta. 2016;936:130-8.

34. Smith CA, Want EJ, O'Maille G, Abagyan R, Siuzdak G, Smith $\mathrm{CA}$, et al. XCMS: processing mass spectrometry data for metabolite profiling using Nonlinear Peak Alignment,Matching, and Identification. Anal Chem. 2006;78(3):779-87.

35. Kuhl C, Tautenhahn R, Böttcher C, Larson TR, Neumann S. CAMERA: An Integrated Strategy for Compound Spectra Extraction and Annotation of Liquid Chromatography/Mass Spectrometry Data Sets. Anal Chem. 2012;84(1):283-9.

36. Sumner LW, Amberg A, Barrett D, Beale MH, Beger R, Daykin $\mathrm{CA}$, et al. Proposed minimum reporting standards for chemical analysis. Metabolomics. 2007;3(3):211-21.

37. Jenke D, Poss M, Sadain S, Story J, Smith W, Reiber D. Identification of caprolactam oligomers and related compounds in aqueous extracts of nylon-6. J Appl Polym Sci. Inc. 2005;95(5): 1262-74.

38. Úbeda S, Aznar M, Vera P, Nerín C, Henríquez L, Taborda L, et al. Overall and specific migration from multilayer high barrier food contact materials - kinetic study of cyclic polyester oligomers migration. Food Addit Contam Part A. 2017;12:1-11. 